\title{
Experimental and Computational Investigation of Flow Fields using Accelerated Erosion Test Ring (AETR)
}

\section{DOI : 10.36909/jer.ICCEMME.15599}

\author{
Vineet Singh", Vinod Singh Yadav**, Vinod Kumar Yadav***, Niraj Kumar*, Manoj Kumar \\ Singh*, Anurag Maheswari", OM Prakash Upadhyay* $^{*}$ \\ *Department of Mechanical Engineering, F.E.T., M.J.P. Rohilkhand University, Bareilly, India. \\ *** Department of Mechanical Engineering, NIT, Uttarakhand, India. \\ **** Department of Mechanical Engineering, GLBITM Greater Noida, India. \\ ***Email: yadavvinod2004@gmail.com; Corresponding Author.
}

\begin{abstract}
The erosion of the blades of hydraulic pumps and turbines, caused due to the water that has enormous suspended particles and erosive agents, is a severe global challenge among scientists and engineers. In the present work, an attempt has been made to compute the extent of erosion caused by water when it passes through devices like hydraulic machines, centrifugal pumps and turbines. Experimental and computational techniques using pitched turbine blades with $45^{\circ}$ with the horizontal plane under down pumping condition is employed for investigation of the suspension phenomena for calculating erosion wear by sand suspended in water in river, canal on turbine, pump blades. An experimental set up, named as Accelerated Erosion Test Rig (AETR), is developed through dimensional analysis. For experimental analysis, the sand particle size and propeller dimensions were varied while running the propeller at different speeds. Experimental results revealed that the propeller speed must be maintained at an optimum value, preferably lower speeds, to ensure maximum lifting of impurities and sand particles from the base of the cylinder.
\end{abstract}

Keywords: Erosion; Pot testing; Propeller; Sand concentration; Suspension speed 


\section{INTRODUCTION}

Turbomachinery's, running at very high rotor speeds, are widely used in modern day industries, in multiphase flow situations. Many researchers have worked over the erosion-wear characteristics of these turbomachines under the influence of solid particles. Several simulation techniques were also used to predict the solid particle behavior during erosion-wear of turbomachines. (Gandhi et al., 2001) experimentally examined the effect of slurry flow, with three different particle sizes, on the performance of two centrifugal pumps and found that the efficiency ratios and head of these pumps are greatly affected by the particle size distribution and solid particle concentration. (Liu et al., 2007) numerically simulated the turbulence, velocity and solid concentration for stirred vessel fitted with a TXL impeller and a single baffle of Rushton turbine and a PTB-6 turbine using Gidaspow model. (ELsawy et al., 2017) studied the effect of adding silicon, chromium and manganese on the microstructures and wear properties of grey cast iron that is used for the design of rollers in sugar industries. They reported that the critical speed of TXL impeller is more than PTB-6 turbine and Rushton turbine. (Wang et al., 2003) experimentally and numerically studied the effects of solid particle concentration and flow field for solid-liquid stirred vessel equipped with Rushton disc impeller and found that the solid phase distribution is more homogeneous at high speeds. (Desale et al., 2004) experimentally tested the erosion-wear behavior of solid-liquid mixture at normal impact in a slurry pot tester using copper, brass, aluminium alloy, and mild steel materials. They observed that the rate of wear, when done at normal impact, strongly depends on hardness ratio of target material and erodent. (Clark, 2002) studied the impact angle, velocity and impact frequency of particles using slurry flow system and reported that his analysis and experimental results are effective tools for the analysis of erosion-corrosion phenomena in wastes. (Minemura and Zhong, 1995) traced the movement of individual solid particle over pump wall casing and reported that the erosion-wear is unaffected due to mutual collision or spinning of the particles. (Rayan et al., 1989) studied the erosive wear characteristics of slurry carried by centrifugal pumps, using weighing method, and reported that the erosion-wear rate of the slurry pumps is directly proportional to the velocity of flow and varies with the transport concentration. (Haimann et al., 2014) presented an integrated suspended sediment monitoring strategy and proved that the suspended sediment transport's temporal variability can be determined more precisely compared with other available methods. (Ridgway et al., 2009) studied the seal wear characteristics of slurry pump gland 
and reported that the particle properties like relative hardness, fracture toughness and size of the shaft sleeve get affected by the particle inorganic chemistry. (Khazam and Kresta, 2009) used three impellers of two diameters and compared the full baffle configuration with two partial height baffle arrangements and found that the performance of surface baffles is superior to full or half baffled configurations. (Thorpe and Stevenson, 2003) studied the mechanism of suspension of particles in pipes and stirred vessels and reported that the shear stresses, due to the suspension of particles, are comparable in both cases. (Gupta et al. , 1995) used pot tester and conducted experiments to analyze the effect of sand concentration, particle size and velocity of fluid. They proposed two sets of correlations that are most suited to predict the erosion-wear of mild steel and brass as pipe materials. (Wood and Jones, 2003) experimentally examined the upstream flows with $10 \%$ water-solid mixture concentration at mean fluid velocity of $3 \mathrm{~m} / \mathrm{s}$. They validated their experimental results with CFD code and reported that the test sand gets degraded in steel pipes and hence needs frequent replacement. (Gohel et al., 2012) modeled solid suspension in stirred tank using CFD software and determined that the turbulent dispersion force and cloud height. (Huang et al., 2008) numerically studied erratic two-phase flow and oil-water mixing phenomena in stirred tank and reported that due to the action of centrifugal force, heavier fluid moves outward and reach the stirred tank's wall. (Ibrahim et al., 2015) studied the effect of solid loading, particle size and clearance of the impeller on the suspension in stirred tank and concluded that with less clearance, radial impellers are superior to downward axial impellers while handling solid-liquid suspension. (Wang et al., 2012) determined the power required to suspend the water-solid slurries in mechanically agitated tanks and found that the power consumption for the solid dispersion can be significantly reduced by removing the baffle plates inside the agitators. (Itoh et al., 2018) developed a hydraulic model test-rig to study the effect of proliferation of sediments and flow during floods due to infringement of natural landslides during hilly torrents. Their results revealed that the depth of the flow is significantly affected by changing the width of flow and the curvature channel and is unaffected by the propagation velocity of a flash flood. (Zhong and Minemura, 1996) used Bitter's model of erosion and studied the erosion damaging behavior of wear-resistant materials and concluded that with an increase in particle diameter, erosion becomes dominant. In the present work, the characteristic of solid-liquid suspension (containing sand) is experimentally studied using a transparent cylinder of $300 \mathrm{~mm}$ diameter and $225 \mathrm{~mm}$ height. The experimental results 
are validated and compared with numerical results using ANSYS Fluent ${ }^{\circledR}$ computational fluid dynamics (CFD) software package.

\section{Materials and methodology}

\section{Details of experimental set up}

To understand the characteristics of solid-liquid suspension experimentally, an experimental set up known as Accelerated Erosion Test Rig (AETR), is developed through dimensional analysis (Fig. 1), is used. To develop AETR set up, a transparent cylinder, made up of acrylic, with a diameter and height of $300 \mathrm{~mm}$ and $225 \mathrm{~mm}$ respectively is used.

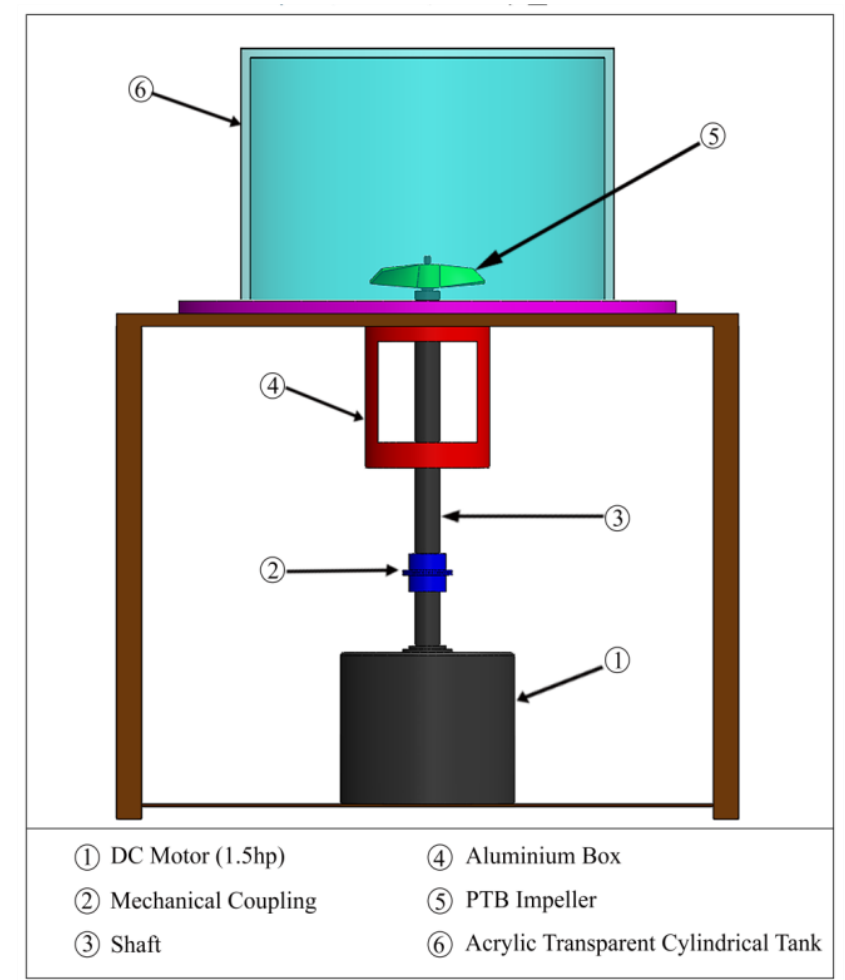

Fig. 1 Experimental facility

The tank, made up of transparent acrylic material, is fitted with four baffle plates (30 $\mathrm{mm} \times 10 \mathrm{~mm}$ ) to ensure proper mixing of the solid-liquid suspension. A shaft is fitted at the bottom side to support the propeller. To prevent any sort of leakage of prepared suspension, an oil seal is fitted at the base of the tank. A $1.5 \mathrm{hp} \mathrm{DC}$ motor, equipped with a speed regulator, is provided to vary the propeller speed as per the requirements. The power from the motor to the propeller shaft is transmitted using a mechanical coupling. Two propellers of different specifications are used for experimentation. The detailed settings to run experiments using two propellers named as Propeller-1 (Prop-1) and Propeller-2 (Prop-2) along 
with other parameters are presented in Table 1. The diameter, width and thickness (in mm) of Prop-1 and Prop- 2 are 140 x 25 × 2 and 150 x 15 × 2 respectively.

Table 1: Particle size and suspension speeds of Prop-1 and Prop-2 for experimental study

\begin{tabular}{|c|c|c|c|c|c|c|}
\hline \multirow{3}{*}{$\begin{array}{l}\text { Sl. } \\
\text { No. }\end{array}$} & \multirow{3}{*}{$\begin{array}{c}\text { Particle } \\
\text { diameter } \\
(\mu \mathrm{m})\end{array}$} & \multirow{3}{*}{$\begin{array}{c}\text { Solid } \\
\text { concentration } \\
\text { (percentage by } \\
\text { weight) }\end{array}$} & \multirow{3}{*}{$\begin{array}{l}\text { Suspension } \\
\text { speed (rpm) }\end{array}$} & \multicolumn{2}{|c|}{ Experimental observations } & \multirow{3}{*}{$\begin{array}{l}\text { suspension } \\
\text { speed } \\
\text { difference } \\
(\mathbf{r p m})\end{array}$} \\
\hline & & & & & & \\
\hline & & & & $\begin{array}{c}\text { Prop-1 } \\
\text { Suspension } \\
\text { speed (rpm) }\end{array}$ & $\begin{array}{c}\text { Prop-2 } \\
\text { Suspension } \\
\text { speed (rpm) }\end{array}$ & \\
\hline 1 & 250 & 1 & 211 & 230 & 210 & 20 \\
\hline 2 & 250 & 2 & 241 & 260 & 230 & 30 \\
\hline 3 & 250 & 3 & 260 & 280 & 240 & 20 \\
\hline 4 & 250 & 5 & 286 & 310 & & \\
\hline 5 & 355 & 1 & 229 & 260 & & \\
\hline 6 & 355 & 2 & 252 & - & - & \\
\hline 7 & 355 & 3 & 272 & - & - & \\
\hline 8 & 355 & 5 & 300 & - & - & \\
\hline 9 & 425 & 1 & 226 & 270 & & \\
\hline 10 & 425 & 2 & 258 & - & - & \\
\hline 11 & 425 & 3 & 279 & - & - & \\
\hline 12 & 425 & 5 & 307 & - & - & \\
\hline
\end{tabular}

\section{Parameters and boundary conditions for computational analysis}

The solid-liquid suspension mixture (containing sand) dealt in the present work is numerically simulated using ANSYS Fluent ${ }^{\circledR}$ computational fluid dynamics (CFD) software package. The suspension mixtures are modeled in ANSYS Fluent ${ }^{\circledR}$ CFD software by solving the conservation equations of mass and momentum in two-dimensional cylindrical coordinates. Due to the rotational symmetry of the cylinder, an axisymmetric model was prepared. For simulations, the flow was assumed to be incompressible, and $\mathrm{k}-\varepsilon$ model was adopted for turbulence modeling. The flow inside the tank is set as turbulent and no-slip boundary condition was applied over the walls. The inertial forces caused due to the fluctuating velocity, dominates over the viscous forces at turbulent flow conditions due to which the viscous heating becomes negligible. Grid independence test was conducted to ensure that the converged solutions are independent of grid size. 


\section{Results and discussions}

The experimental set up, shown in Fig. 1, is used to conduct experiments at different sand concentration levels and with different sand particle size. The effect of varying the particle size and impeller speed on the lifting of the sand particles by the impeller is observed experimentally.

\section{Experimental analysis using Prop-1}

The results of the experimental observations are plotted in Fig. 2 and Fig. 3 for $1 \%$ and $2 \%$ sand concentration (Conc.) respectively and for a constant particle size (Sp) of $250 \mu \mathrm{m}$.

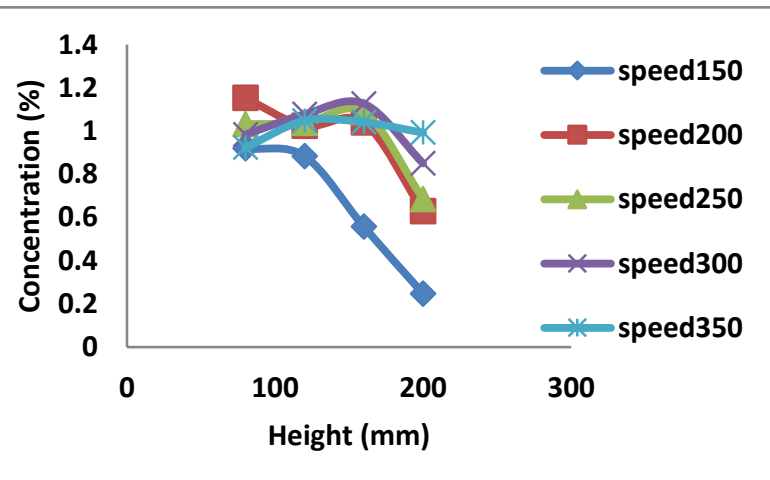

Fig. $2 \mathrm{Sp}=250 \mu \mathrm{m}$ and Conc. $=1 \%$

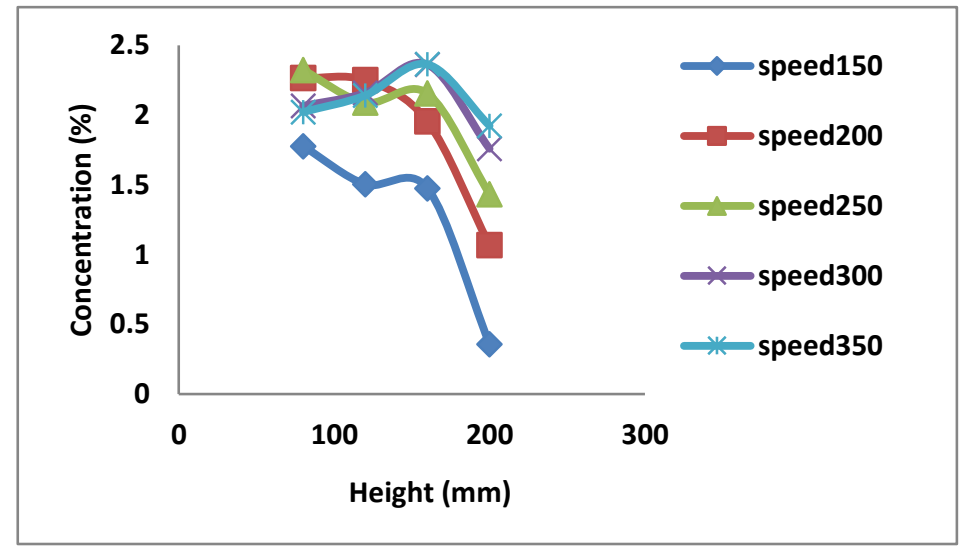

Fig. $3 \mathrm{Sp}=250 \mu \mathrm{m}$ and Conc. $=2 \%$

The $\mathrm{x}$-axis represents the height of the cylinder and $\mathrm{y}$-axis represents the percent sand concentration. It can be observed from Fig. 2 and 3 that the concentration of sand, at $150 \mathrm{rpm}$, is maximum around a height of $120 \mathrm{~mm}$. However, with an increase in speed of the impeller gradually from $150 \mathrm{rpm}$ to 350 rpm, the maximum sand concentration observed is around 1\% (Fig. 1) and 2\% (Fig. 3). Both the plots (Fig. 2 and Fig. 3) indicate a flattening trend of the concentration profile with increasing height. It can also be observed that, no sand concentration is traced beyond a height of $200 \mathrm{~mm}$ with 1-2\% concentration levels. The concentration of sand is then increased to $3 \%$ and $5 \%$ for fixed particle size of $250 \mu \mathrm{m}$ and the results are plotted in Fig. 4 and 5 respectively. 
The sand particle size is then increased to $355 \mu \mathrm{m}$ (Fig. 6) and $425 \mu \mathrm{m}$ (Fig. 7) with fixed concentration of $1 \%$. No sand concentration was seen at $150 \mathrm{rpm}$ for 355 as well as $425 \mu \mathrm{m}$ size sand suspension up to $200 \mathrm{~mm}$ height. This indicates that, with larger size of sand particles, the speed of the impeller must

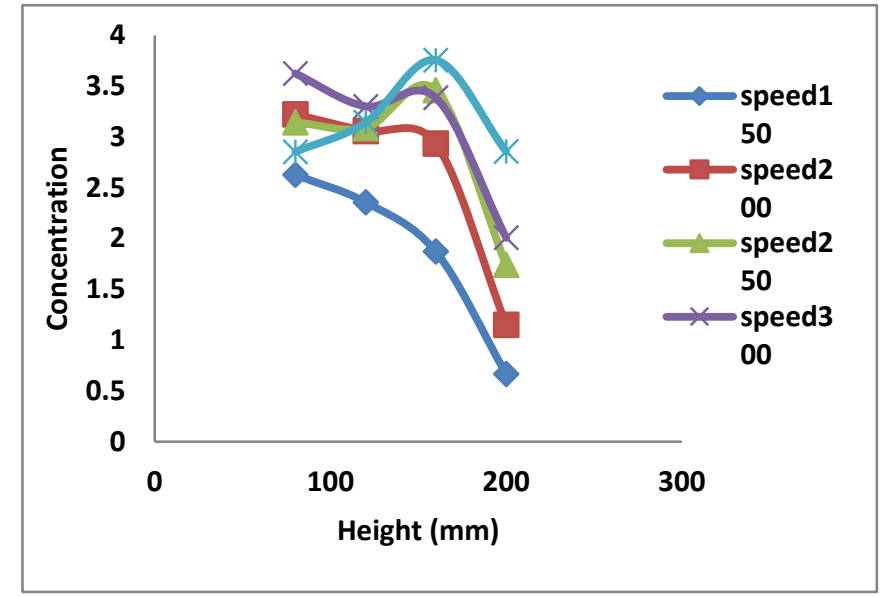

Fig. $4 \mathrm{Sp}=250 \mu \mathrm{m}$ and Conc. $=3 \%$

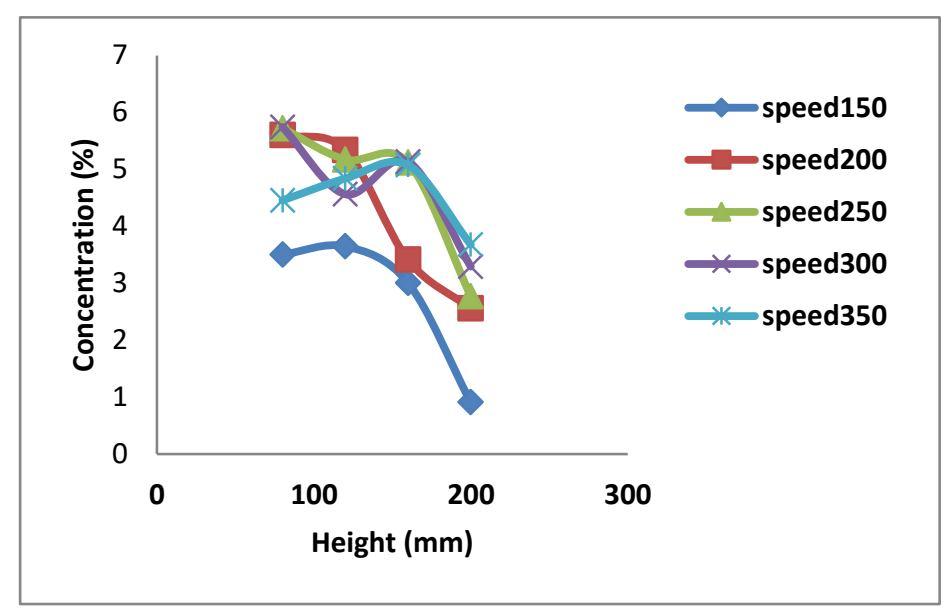

Fig. $5 \mathrm{Sp}=250 \mu \mathrm{m}$ and Conc. $=5 \%$

be increased to ensure the lifting of sand particles.

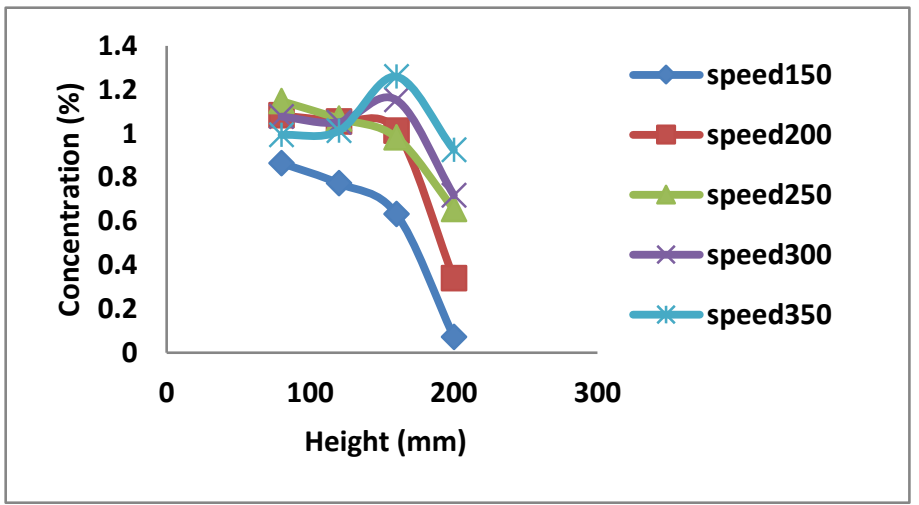

Fig. $6 \mathrm{Sp}=355 \mu \mathrm{m}$ and Conc. $=1 \%$

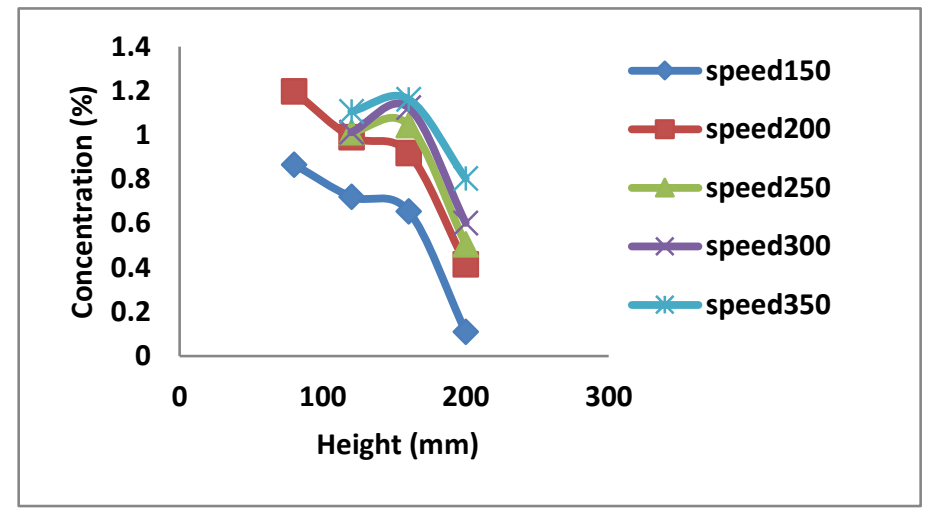

Fig. $7 \mathrm{Sp}=425 \mu \mathrm{m}$ and Conc. $=1 \%$

\section{Computational analysis of solid-liquid suspension}

Sand volume fraction, defined as the ratio of the volume of sand in a mixture of sand and water. To numerically check the observations made during experimentation, a two-dimensional numerical model is developed using ANSYS Fluent CFD software. The numerical model mimicked the cylinder used for experimental analysis. The contours of sand concentration, plotted in Fig. 14, at different heights are 
monitored after convergence of the simulated results. Fluent's post-processing toll is used for plotting the results and extracting the simulated data. The results showed that at $150 \mathrm{rpm}$ and $1 \%$ sand concentration, very less amount of sand is lifted from the base of the cylinder. This indicates that in order to lift entire volume of sand from the base of the cylinder to the upper end, the propeller speed must be increased to create more turbulence at lower end. Also, the distribution of sand at upper end is non-uniform. In addition, it is falling downwards under the influence of body forces. The contours of velocity and pressure fields are plotted in Fig. 9 and 10 respectively to understand the behavior of flow pattern with $1 \%$ sand concentration.

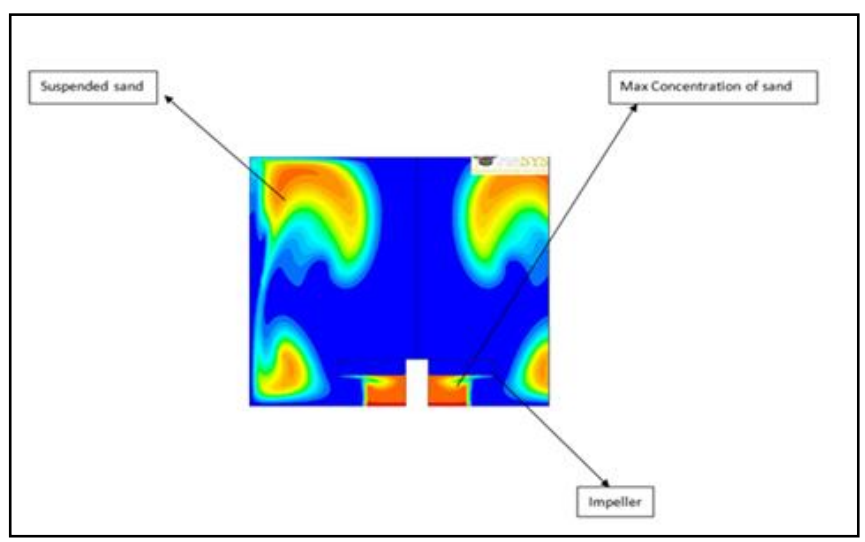

Fig. 8 Numerical results with $1 \%$ sand concentration at 150 rpm

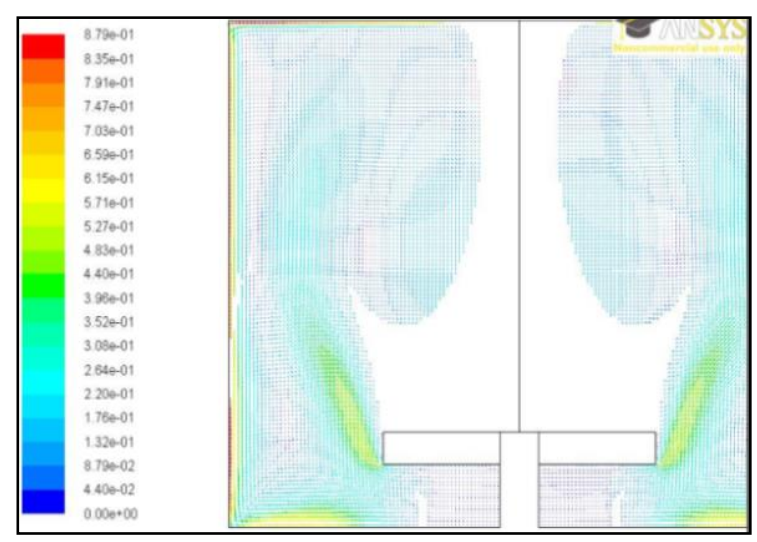

Fig. 9 Velocity profile at $150 \mathrm{rpm}$ and $1 \%$ Conc.

As, expected, the sand particle velocity is higher at the immediate vicinity of the impeller (Fig. 9) and consequently, the pressure is lower at the bottom of the impeller and high at the top of the impeller (Fig. 10). Due to this, the suspended sand particles travel from bottom of the cylinder to top side and then falls down under the action of gravity. The path lines were also traced to understand the phenomena of sand particle travel. As can be seen from Fig. 11, the path lines with $1 \%$ sand particle concentration (yellow colored), show that the sand falls downwards due to the effects discussed in context to Figures 8-10. At the base of the propeller, the settled sand is shown by blue lines. 


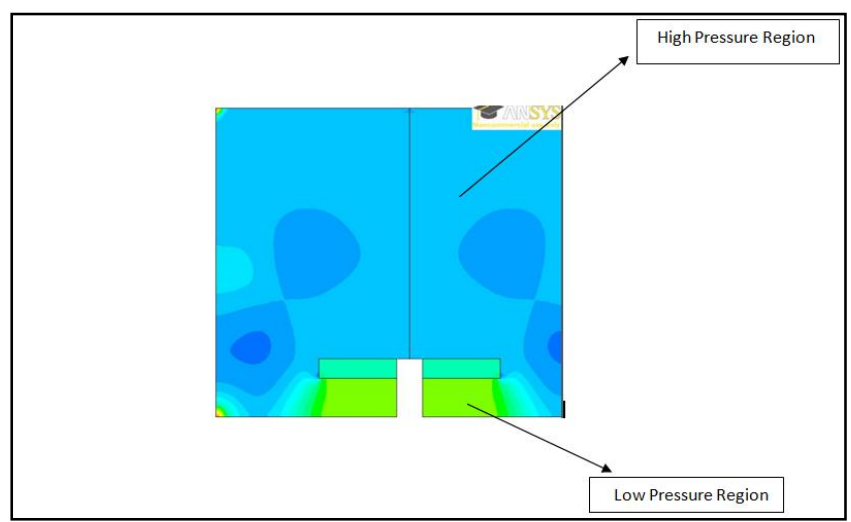

Fig. 10 Pressure field at $150 \mathrm{rpm}$ and $1 \%$ Conc.

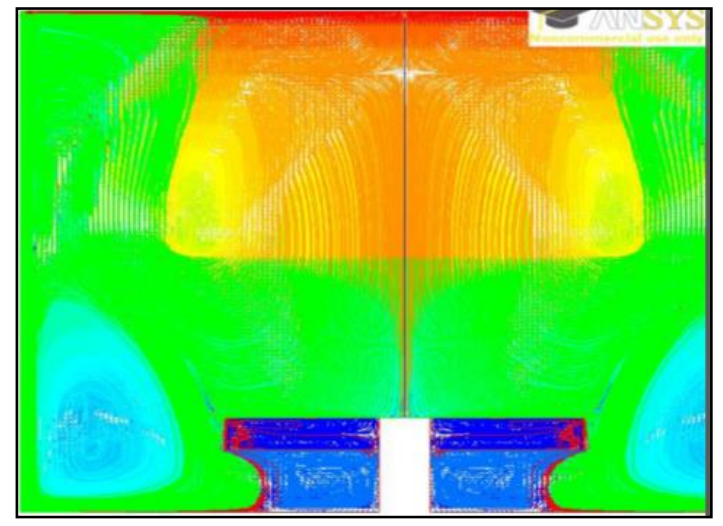

Fig. 11 Path lines at $150 \mathrm{rpm}$ and $1 \%$ sand Conc.

To study the effect of varying the concentration of sand particles on its lifting phenomena, twodimensional numerical models, identical to the specifications of Prop-1, is prepared. After grid independence test, numerical results were computed by varying the sand concentration from 3-5\%. Fig. 12 and Fig. 13 shows the results computed for same particle size and sand concentrations of $1 \%$ and $2 \%$ respectively. It was observed that, at an impeller speed of $150 \mathrm{rpm}$ (Fig. 12), a good amount of sand

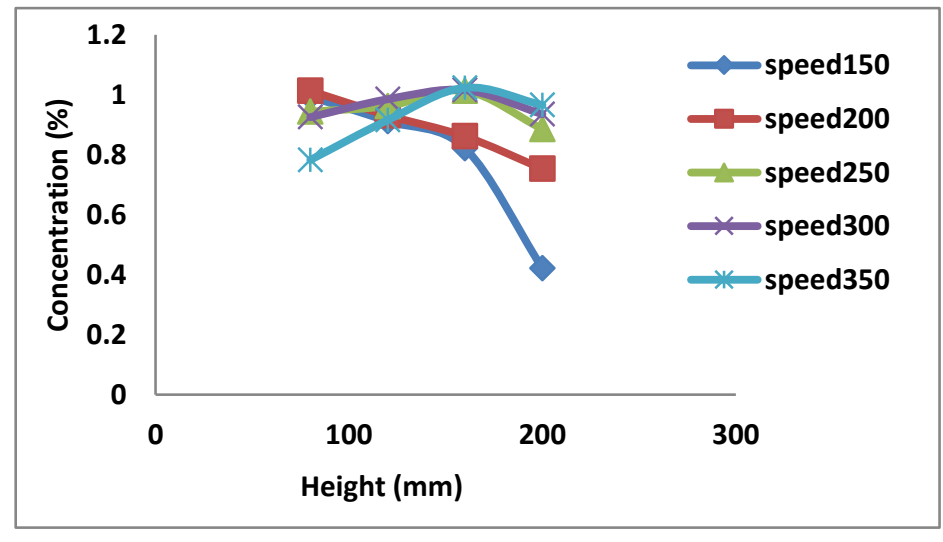

Fig. $12 \mathrm{Sp}=250 \mu \mathrm{m}$ and Conc. $=1 \%$

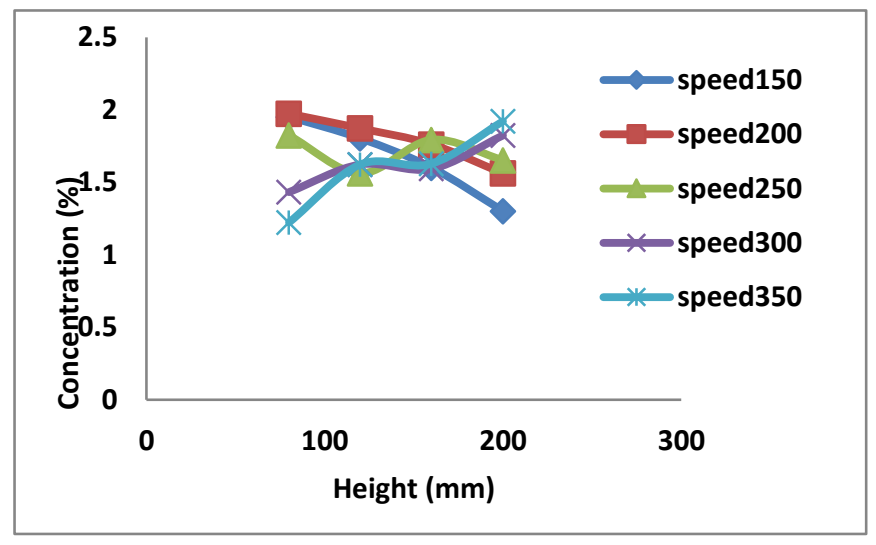

Fig. $13 \mathrm{Sp}=250 \mu \mathrm{m}$ and Conc. $=2 \%$.

particles can be located at a height of $120 \mathrm{~mm}$. In addition, at a height of around $200 \mathrm{~mm}$, the concentration of sand is negligibly small. On the other hand, when the speed of the impeller is increased from $150 \mathrm{rpm}$ to $350 \mathrm{rpm}$, the concentration of sand is noticed to be around $1 \%$ inside the cylinder. Similar trends were observed for sand concentration of $2 \%$ (Fig. 13).

Now, the sand concentration is varied in the range of 3-5\% and the results are plotted in Fig. 14 and 15. 
It can be observed that at an impeller speed of $150 \mathrm{rpm}$, and with 3-5\% sand concentration, small amount of sand can be located even at a height of $200 \mathrm{~mm}$. This further supports that, with an increased sand concentration, the suspension speed may compulsorily be increased. In addition, the maximum sand concentration lies in between 100- and 200-mm height in all cases.

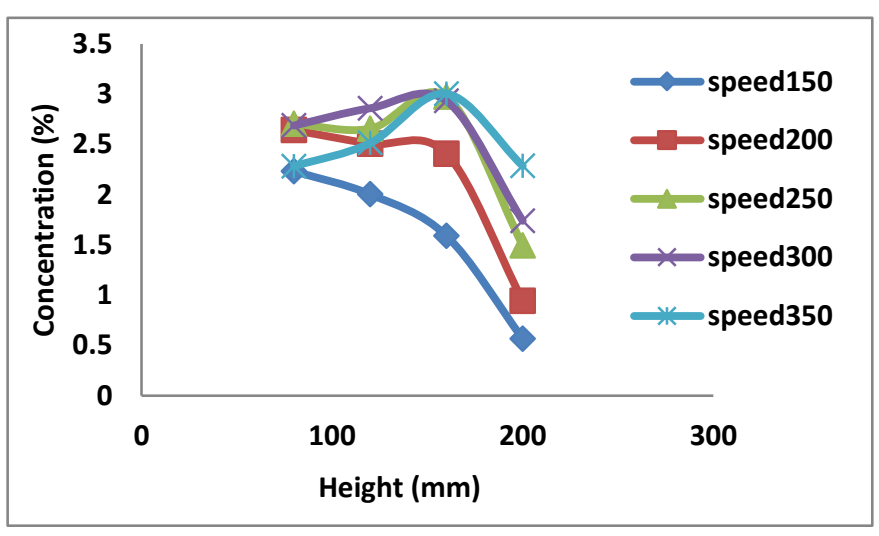

Fig. $14 \mathrm{Sp}=250 \mu \mathrm{m}$ and Conc. $=3 \%$

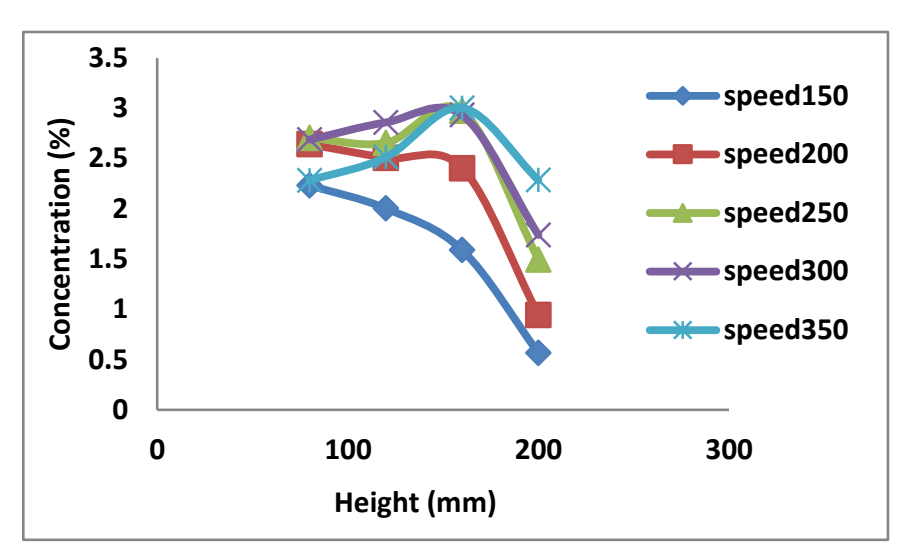

Fig. $15 \mathrm{Sp}=250 \mu \mathrm{m}$ and Conc. $=5 \%$.

Fig. 16 and Fig. 17 compare the numerically simulated results with particle size of $355 \mu \mathrm{m}$ and 425 $\mu \mathrm{m}$ respectively with fixed concentration of $1 \%$.

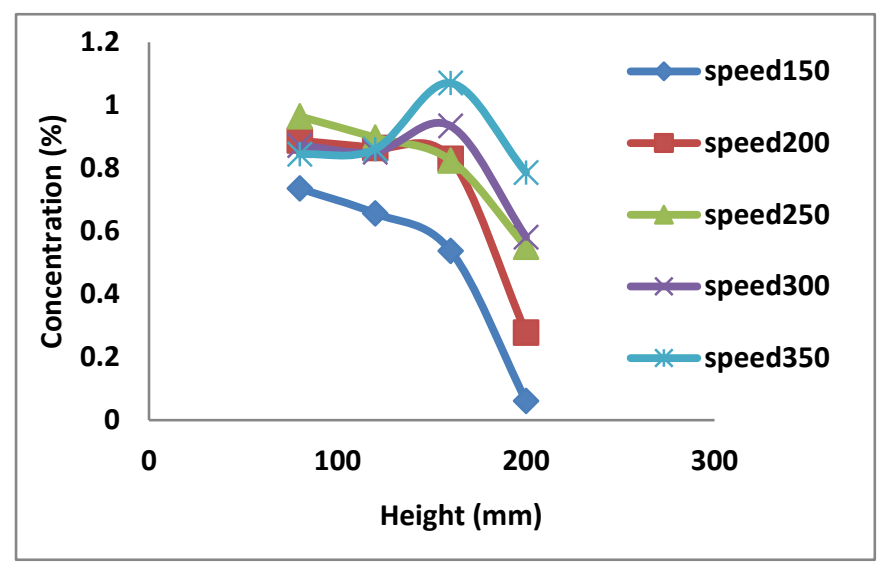

Fig. $16 \mathrm{Sp}=355 \mu \mathrm{m}$ and Conc. $=1 \%$

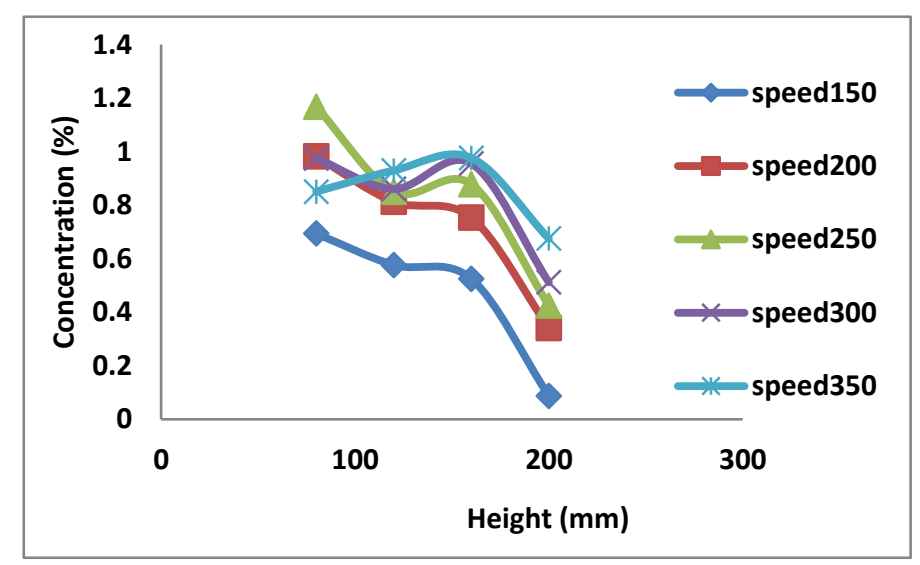

Fig. $17 \mathrm{Sp}=425 \mu \mathrm{m}$ and Conc. $=1 \%$.

\section{Validation of experimental and numerical results}

Figures 18-22 presents a quantitative comparison of results that were obtained using experimentation and numerical simulations. A difference of about 12 to $20 \%$ in numerically simulated results as compared to experimentally investigated results was observed. This may be attributed to the fact that in 
computational simulations, several physical factors cannot be realistically modeled due to the limitation of the model. Furthermore, it has been tried to maintain the exact concentration of sand during experimentation. But, in actual practice, there may be some other impurities inside the impellers that may exhibit an increased concentration of suspended particles as observed in some of the experimental results. The set was cleaned after every experimental procedure to ensure that the traces of previous experiments have been removed. Some of the experiments were repeated to check the accuracy of measurements.

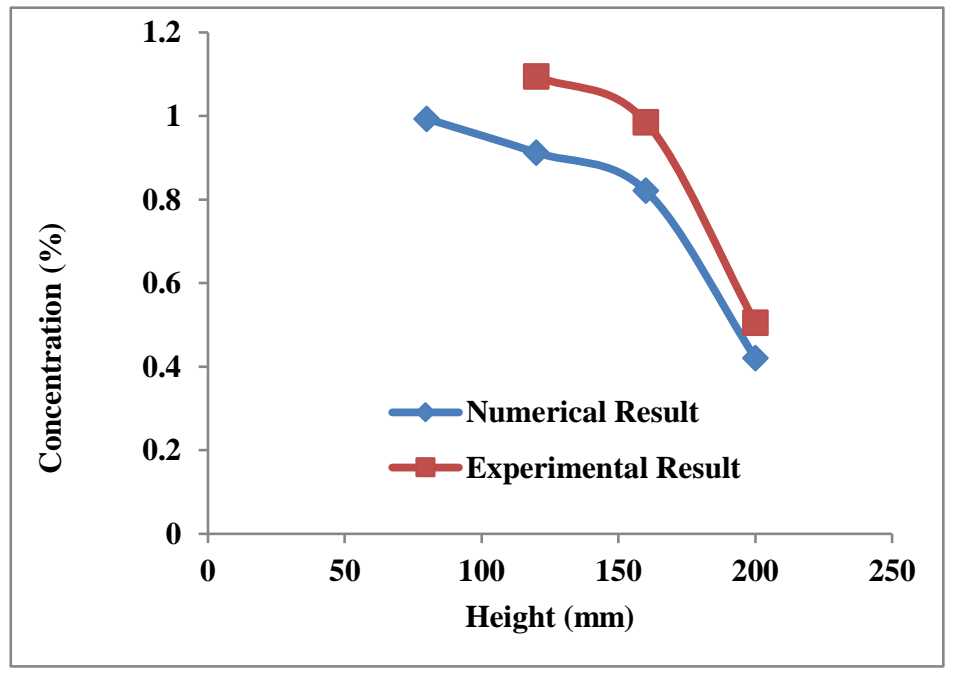

Fig. $16 \mathrm{Sp}=250 \mu \mathrm{m}$ and Conc. $=1 \%$

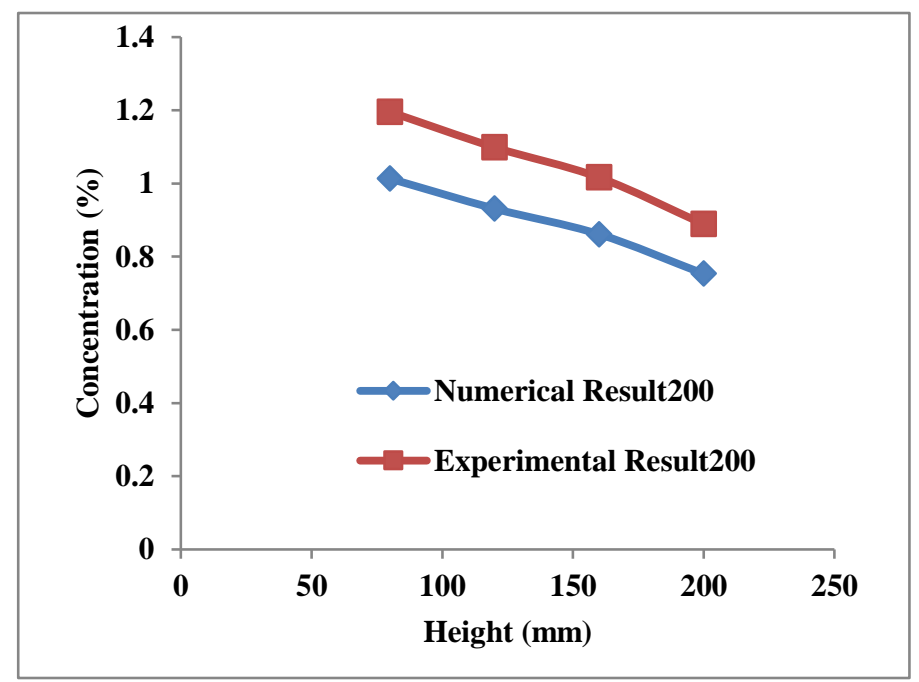

Fig. $17 \mathrm{Sp}=250 \mu \mathrm{m}$ and Conc. $=2 \%$. 


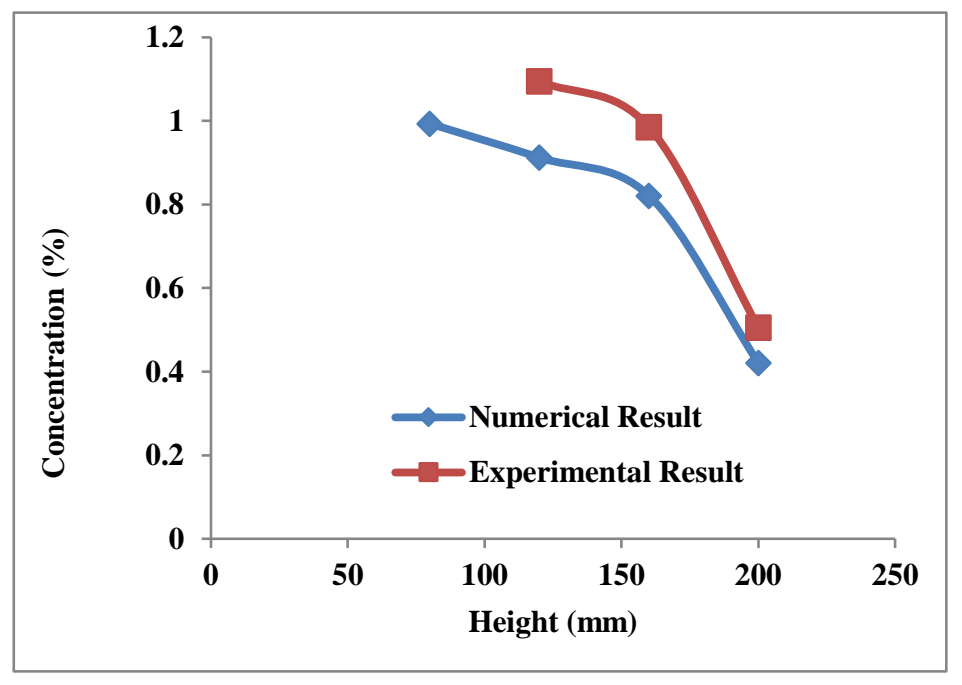

Fig. $18 \mathrm{Sp}=250 \mu \mathrm{m}$ and Conc. $=3 \%$

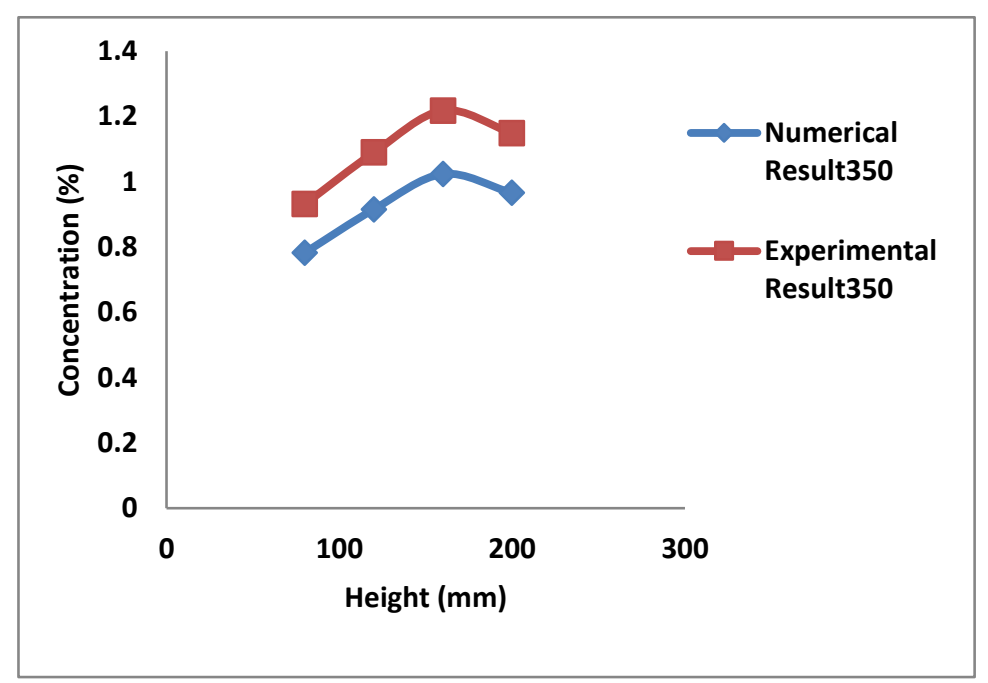

Fig. $20 \mathrm{Sp}=255 \mu \mathrm{m}$ and Conc. $=1 \%$

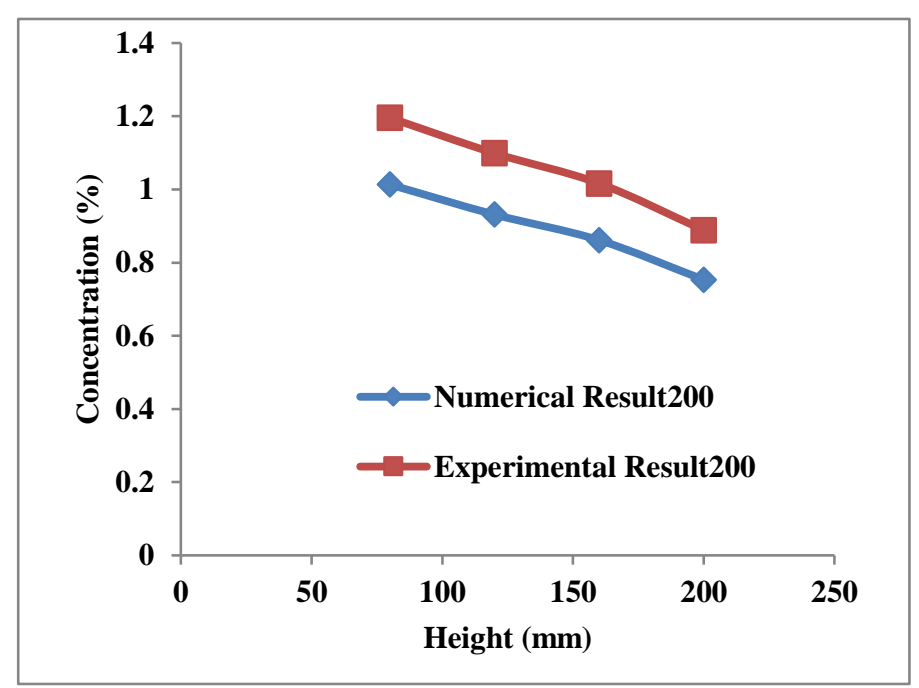

Fig. $19 \mathrm{Sp}=250 \mu \mathrm{m}$ and Conc. $=5 \%$.

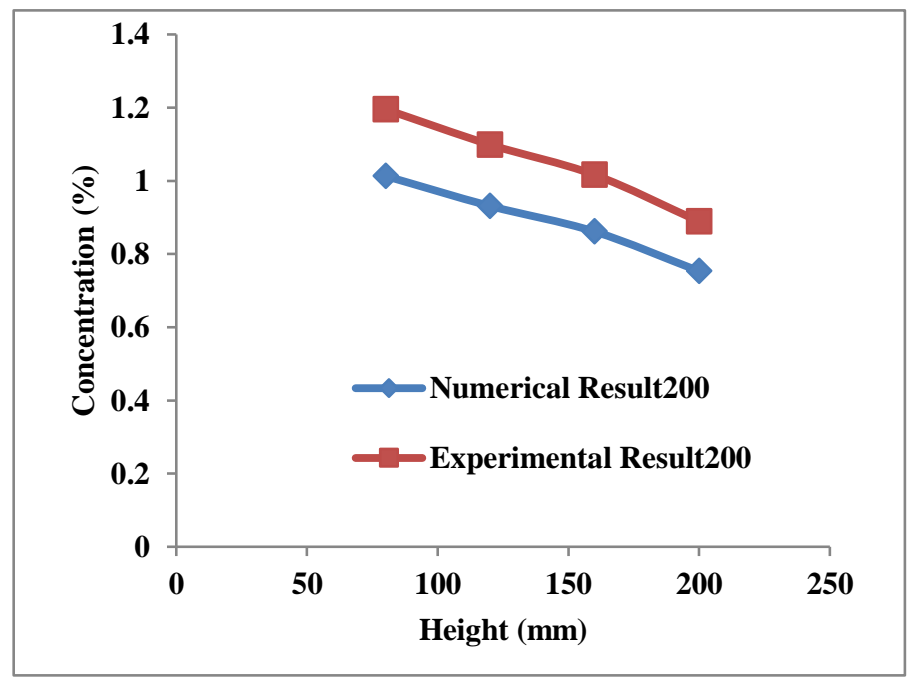

Fig. $21 \mathrm{Sp}=425 \mu \mathrm{m}$ and Conc. $=1 \%$. 


\section{Conclusions}

In present work, an experimental set up, named as AERT, to study the suspension physiognomies of solid-liquid mixture, is developed. The effects of impeller diameter, speed and particle size on the suspension speed of the mixtures were investigated experimentally. A two-dimensional numerical model of propellers used in the experimental analysis was also prepared to get an insight of the pressure, velocity and flow paths of such mixtures. The following conclusions were drawn from the experimental and numerical results:

1- The suspension speed is directly proportional to the sand concentration and particle size of the sand.

2- The size of the propeller does not affect the suspension speed significantly. An optimum size (diameter) of the propeller is sufficient to handle solid-liquid mixture studied in this work.

\section{References}

Gandhi, B. K., Singh, S. N., \& Seshadri, V. (2001). Performance characteristics of centrifugal slurry pumps. J. Fluids Eng., 123(2): 271-280.

Liu, B., Zheng, Y., Chen, M., Chen, X., \& Jin, Z. (2017). CFD simulation of the mixing and dispersing of floating particles in a viscous system. Brazilian Journal of Chemical Engineering, 34: 1175-1189.

ELSawy, E. E. T., El-Hebeary, M. R., \& El Mahallawi, I. S. E. (2017). Effect of manganese, silicon and chromium additions on microstructure and wear characteristics of grey cast iron for sugar industries applications. Wear, 390: 113-124.

Wang, F., Wang, W., Wang, Y., \& Mao, Z. (2003, December). CFD simulation of solid-liquid two-phase flow in baffled stirred vessels with Rushton impellers. In Proc 3rd Intl Conf on CFD in the Minerals and Process Industries (pp. 287-292). Melbourne Australia.

Desale, G. R., Gandhi, B. K., \& Jain, S. C. (2008). Slurry erosion of ductile materials under normal impact condition. Wear, 264(3-4): 322-330.

Clark, H. M. (2002). Particle velocity and size effects in laboratory slurry erosion measurements OR... do you know what your particles are doing? Tribology International, 35(10): 617624.

Minemura, K., Zhong, Y., \& Uchiyama, T. (1995). Numerical prediction of erosion wear on pump casing under solid-water two-phase flow conditions; Koeki nisoryuji no pump casing mamoryo no suchi yosoku. Nippon Kikai Gakkai Ronbunshu. B Hen (Transactions of the Japan Society of Mechanical Engineers. Part B), 61.

Rayan, M. A., \& Shawky, M. (1989). Evaluation of wear in a centrifugal slurry pump. Proceedings of the Institution of Mechanical Engineers, Part A: Journal of Power Engineering, 203(1): 19-23.

Haimann, M., Liedermann, M., Petra, L. A. L. K., \& Habersack, H. (2014). An integrated suspended sediment transport monitoring and analysis concept. International Journal of Sediment Research, 29(2): 135-148. 
Ridgway, N., Colby, C. B., \& O’Neill, B. K. (2009). Slurry pump gland seal wear. Tribology international, 42(11-12): 1715-1721.

Khazam, O., \& Kresta, S. M. (2009). A novel geometry for solids drawdown in stirred tanks. Chemical Engineering Research and Design, 87(3): 280-290.

Thorpe, R. B., \& Stevenson, P. (2003). Suspension of particles from the bottom of pipes and stirred tanks by gassed and ungassed flows. The Canadian Journal of Chemical Engineering, 81(3-4): 351-359.

Gupta, R., Singh, S. N., \& Sehadri, V. (1995). Prediction of uneven wear in a slurry pipeline on the basis of measurements in a pot tester. Wear, 184(2): 169-178.

Wood, R. J. K., \& Jones, T. F. (2003). Investigations of sand-water induced erosive wear of AISI 304L stainless steel pipes by pilot-scale and laboratory-scale testing. Wear, 255(1-6): 206218.

Gohel, S., Joshi, S., Azhar, M., Horner, M., \& Padron, G. (2012). CFD modeling of solid suspension in a stirred tank: Effect of drag models and turbulent dispersion on cloud height. International Journal of Chemical Engineering, 1-9.

Huang, S., Mohamad, A., \& Nandakumar, K. (2008). Numerical Analysis of a Two-Phase Flow and Mixing Process in a Stirred Tank. International Journal of Chemical Reactor Engineering, 6(1): $1-17$.

Ibrahim, S., Wong, S. D., Baker, I. F., Zamzam, Z., Sato, M., \& Kato, Y. (2015). Influence of geometry and slurry properties on fine particles suspension at high loadings in a stirred vessel. Chemical Engineering Research and Design, 94: 324-336.

Wang, S., Boger, D. V., \& Wu, J. (2012). Energy efficient solids suspension in an agitated vesselwater slurry. Chemical engineering science, 74: 233-243.

Itoh, T., Ikeda, A., Nagayama, T., \& Mizuyama, T. (2018). Hydraulic model tests for propagation of flow and sediment in floods due to breaking of a natural landslide dam during a mountainous torrent. International Journal of Sediment Research, 33(2): 107-116.

Zhong, Y., \& Minemura, K. (1996). Measurement of erosion due to particle impingement and numerical prediction of wear in pump casing. Wear, 199(1): 36-44. 